\title{
Matemática aplicada a enfermagem: cálculo de dosagem e diluição de medicamentos em um portal educacional
}

\author{
Mathematics applied to nursing: calculation of drug dosage \\ and dilution in an educational portal
}

\author{
Adriano dos Santos Cunha' • Claudia Maria Messias² • Ana Cláudia Lopes Almeida ${ }^{3}$
}

\begin{abstract}
RESUMO
O presente trabalho tem como objetivo verificar os principais saberes de Matemática empregados em Enfermagem, bem como a analisar a utilização de um portal de apoio educacional como forma de auxiliar estudantes com cálculo de dosagem e diluição de medicamentos. Foi realizado um estudo comparativo com pré e pós teste baseado em um instrumento composto de oito questões objetivas utilizadas em concursos ou processos públicos de avaliação. $O$ estudo foi realizado com alunos concluintes do curso de graduação em enfermagem da Universidade Castelo Branco uma instituição particular localizada na cidade do Rio de Janeiro, e apresentou um portal online de apoio educacional intitulado "cálculo tem remédio" que foi testado como ferramenta de ensino.Após a realização de pré e pós teste do uso do portal, os dados foram analisados pelo programa estatístico Minitab® - versão 17. Como resultado, foi observada uma diferença significativa $(p<0,05)$ entre as médias do pré-teste e pós-teste, e concluiu que a pesquisa logrou êxito quanto aos objetivos propostos e que o portal de apoio educacional pode contribuir para a formação do profissional de enfermagem.
\end{abstract}

Palavras-chave: enfermagem, matemática; cálculo de dosagem; portal educacional.

\begin{abstract}
The present work aims to verify the main knowledge of Mathematics applied in Nursing, as well as to analyze the use of an educational support portal as a way to assist students with calculation of drug dosage and dilution.A comparative study with pre and post-test was carried out based on an instrument composed of eight objective questions used in contests or public evaluation processes. The study was carried out with graduating students of the nursing undergraduate course at Castelo Branco University, a private institution located in the city of Rio de Janeiro, and presented an online educational support portal titled "calculation has medicine" that was tested as a teaching tool.After the pre and post-test of the portal use, the data were analyzed by the statistical program Minitab® - version I7. As a result, a significant difference $(p<0.05)$ was observed between the means of the pre-test and post-test and concluded that the research was successful in terms of the proposed objectives and that the educational support portal can contribute to the training of the nursing professional. Keywords: nursing, mathematics; calculation of dosage; educational portal.
\end{abstract}

\footnotetext{
'Estudo baseado em Dissertação de Mestrado de Adriano dos Santos Cunha intitulada "Matemática Aplicada A Enfermagem: apoio ao ensino do cálculo de dosagem e diluição de medicamentos através de um portal educacional." Apresentada a Universidade Severino Sombra,Vassouras/RJ 20 I4.

${ }^{2}$ Mestre em Educação Matemática, Especialista em Docência do Ensino Superior, Especialista em Planejamento, Implementação e Gestão de cursos em EAD, Graduado em Ciências com habilitação plena em Matemática, Professor da Universidade Castelo Branco RJ.profadrianocunha@hotmail.com

${ }^{3}$ Doutora em ciências da enfermagem Professora auxiliar da Universidade Castelo Branco e Enfermeira do HMMC.

${ }^{4}$ Mestre em enfermagem pela Escola de Enfermagem Anna Nery - UFRJ, Pós-graduação em Enfermagem em Alta Complexidade pela faculdade de enfermagem da UERJ.
} 


\section{INTRODUÇÃO}

As teorias e práticas associadas à informática na educação vêm repercutindo em nível mundial, justamente porque as ferramentas e mídias digitais oferecem à didática objetos, espaços e instrumentos capazes de renovar as situações de interação, expressão, criação, comunicação, informação, e colaboração, tornando-a muito diferente daquela tradicionalmente fundamentada na escrita e nos meios impressos ${ }^{(1)}$.

A educação brasileira e a mundial vêm incorporando novas intervenções nestes mais recentes dez anos, no tocante à presença e implementação de tecnologias recentes na educação.

No ensino para a área de Saúde, é notável a responsabilidade dos professores de matemática, com destaque especial para o pessoal de enfermagem, porque é através do domínio de cálculos matemáticos básicos que o futuro profissional resolverá cálculos de dosagem que envolvem o preparo de dosagem de medicamentos. Esses profissionais têm sempre a vida de pessoas em suas mãos e devem desempenhar seu trabalho com responsabilidade e competência ${ }^{(2)}$.

Associando a atividade do profissional de enfermagem aos conteúdos de Matemática, alguns desses conhecimentos são necessários na prática em enfermagem e os que apresentam um maior grau de dificuldade são os relacionados às operações que necessitam de divisão, como porcentagem e números fracionários. Essas operações são muito utilizadas em cálculos de gotejamento de soro, preparo e diluição de medicamentos, alterações na concentração de algumas drogas e preparo de soluções ${ }^{(3)}$.

Algumas prescrições, baseadas em Unidades Internacionais de Medidas, nem sempre coincidem com a apresentação da droga em si, já que existem muitas variações nas apresentações, de acordo com cada fabricante. Outras vezes, a forma de apresentação diverge da prescrita, necessitando de conversão de unidades de medida, bem como diluição ou adequação na concentração e rediluição dos medicamentos prescritos. $\bigcirc$ tema cálculo de dosagem e diluição de medicamentos abordado nos cursos de enfermagem também requer conhecimento de conceitos básicos da matemática, como as quatro operações fundamentais, unidades de medidas, porcentagem e, principalmente, regra de três.

Trabalhar com números é uma competência cognitiva necessária ao profissional da área da saúde, que esbarra no déficit de aprendizagem que muitos enfrentaram no ensino fundamental e médio, portanto é um desafio para quem conduz o treinamento tornar a atividade fácil e interessante, daí a importância de se utilizar técnicas didáticas que possibilitem o aprendizado ${ }^{(4)}$. $O$ preparo de medicamentos nas instituições hospitalares é de responsabilidade legal da equipe de enfermagem. Sendo assim, é necessário que o profissional que os prepara esteja consciente e seguro de sua ação(3).

Faz-se necessário, portanto, que a formação em enfermagem, ainda durante as atividades pedagógicas na universidade, seja o espaço de referência que os estudantes necessitam para enfrentar com agilidade, competência e sabedoria as questões que possam envolver um erro durante o cuidado de enfermagem, assegurando assim ao paciente, à família, à instituição de saúde e ao próprio profissional a segurança e qualidade que tanto almejam ${ }^{(5)}$

O presente estudo trata das habilidades matemáticas desenvolvidas, aplicadas ao conhecimento do cálculo de medicamentos e tem como objetivos verificar e analisar a eficácia de um portal de apoio educacional para discentes de enfermagem na prática do cálculo de dosagem de medicamentos.

Sabe-se que as tecnologias educativas são necessárias e relevantes, pois são capazes de fornecer informações que melhoram o conhecimento e o enfrentamento do profissional ${ }^{(6-7)}$.

\section{MATERIAL E MÉTODOS DESCRIÇÃO DO PRODUTO}

De modo geral, um portal educacional refere-se ao uso de recursos digitais de comunicação, principalmente através de softwares educacionais via web que reúnem diversas ferramentas de interação( ${ }^{(8)}$.

O portal de apoio educacional foi desenvolvido como produto de dissertação relacionada ao Mestrado Profissional em Educação Matemática da Universidade Severino Sombra (USS), o projeto foi apresentado aos alunos concluintes do curso de graduação em Sistemas de Informação da mesma universidade e voluntariamente dois desses alunos aderiram ao projeto e realizaram seus trabalhos de conclusão de curso baseados na mesma pesquisa. O portal está disponível na rede mundial de computadores - internetno endereço eletrônico www. calculotemremedio.com.br.

O portal disponibiliza, em sua tela principal, cinco opções com os recursos disponíveis:

- Botão Ferramentas - encontram-se a calculadora e a ferramenta para cálculo de regra de três. Essas ferramentas foram desenvolvidas a fim de proporcionar interatividade e ensino.

- Apostilas - estão disponíveis três apostilas com o objetivo de auxiliar o usuário quanto a fundamentação, com exemplos.

- Vídeo Aulas - três aulas sobre regra de três simples, onde o usuário poderá acompanhar como organizar e determinar o valor da incógnita.

- Banco de Questões - está disponível no portal uma lista de exercícios com questões que fizeram parte de provas de concursos públicos, exames 
nacionais e também questões elaboradas pelo autor desse estudo.

- Tutorial - é uma ferramenta de ensino e aprendizagem, tem por objetivo auxiliar o entendimento do portal educacional mostrando o produto e facilitando processo de aprendizagem.

Uma das opções do portal educacional é a ferramenta para auxílio no cálculo de regra de três simples, a Figura 2 mostra a tela inicial onde o usuário poderá selecionar as grandezas, inserir os valores, observar a equação e, quando clicar no botão calcular, obter o valor da variável.

São disponibilizadas no portal educacional duas calculadoras. Uma delas possui a finalidade de auxiliar no ensino de cálculo de diluição e dosagem de medicamentos, como mostra a Figura 3.

A outra calculadora disponibilizada no portal auxilia no cálculo de gotejamento, onde a principal função é controlar o volume e o tempo que as soluções devem ser assimiladas. $\mathrm{Na}$ Figura 4 pode ser visualizada a tela inicial da calculadora de medicamentos.

\section{METODOLOGIA}

O estudo foi realizado no ano de 2014 na Universidade Castelo Branco, localizada no bairro de Realengo, Zona Oeste da cidade do Rio de Janeiro. Foi conduzido um estudo experimental dividido em dois momentos de participação.

O primeiro momento, realizado no mês de julho de 20I4, chamado de pré-teste contou com a participação de 20 discentes selecionados de forma aleatória, concluintes do curso de Enfermagem. Foi utilizado um instrumento de coleta de dados composto de oito questões objetivas sobre cálculo de medicação utilizadas em concursos ou processos públicos de avaliação. O critério de avaliação do instrumento foi o percentual de acertos.

O segundo momento, chamado de pós-teste, foi realizado 30 dias após a aplicação do pré-teste, ocorreu no mês de Agosto de 2014 e contou com a participação de 18 discentes, pois um participante se evadiu da instituição e outro encontrava-se em licença médica. $O$ portal foi apresentado e orientado aos discentes que se ambientassem com a ferramenta e após resolvessem, no mínimo, três questões por semana e que permanecessem ao menos 30 minutos no ambiente explorando e criando situações a serem resolvidas.

A fim de testar a eficácia do portal, foi realizada uma análise estatística baseada no percentual de acertos usando dados coletados no pré-teste e em dados obtidos com o pós-teste. Os dados foram analisados utilizando-se o programa estatístico Minitab ${ }^{\circledR}$ - versão $17^{(9)}$.

O estudo foi inscrito na Plataforma Brasil, que é um sistema nacional e unificado de registros de pesquisas envolvendo seres humanos e aprovado pelo Comitê de Ética em Pesquisa da Universidade Severino Sombra sob o CAAE: 36932 I |4.9.0000.5290.

\section{RESULTADOS}

O estudo experimental realizado evidenciou a existência de diferença na aprendizagem significativa com a utilização do portal educacional no processo de ensino e de aprendizagem de cálculo de diluição, dosagem e gotejamento de medicamentos.

Pesquisas apontam que o conhecimento e as dúvidas dos discentes precisam ser considerados na elaboração de tecnologias educativas; soma-se a isso a estreita relação entre a qualidade do material educativo e o uso de princípios e formas definidas de elaboração(10).

A validação de tecnologias apoiadas no impacto na aprendizagem dos discentes é considerada uma estratégia que, mediante o uso sistemático dos conhecimentos disponíveis, visa elaborar uma nova intervenção e melhorar significativamente uma intervenção.

O processo de avaliação consiste em determinar em que medida os objetivos educacionais estão sendo realmente alcançados ${ }^{(11-12)}$.

Estes objetivos produzem modificações desejáveis nos padrões de comportamento do estudante. Portanto, a avaliação deve apreciar o comportamento dos estudantes na fase inicial do processo, e posteriormente em outras, para identificar as mudanças que possam estar acontecendo. Sem saber quais eram as condições iniciais do estudante, não é possível determinar se houve modificações. Um outro marco de avaliação é necessário, também, para se obter uma estimativa da permanência da aprendizagem, que deve ser realizado após algum tempo que o ensino se completou.

A avaliação da aprendizagem permite verificar como o aluno está assimilando os conhecimentos, como isto modifica a sua visão em relação ao mundo, estimula o desenvolvimento do raciocínio e aumenta a sua capacidade de participação na realidade que está vivendo.

Realizada a correção dos instrumentos, as notas dos alunos no pré-teste e pós-teste foram organizadas, como mostra a Tabela I.

Trata-se de duas amostras dependentes, já que os mesmos alunos participaram dos dois grupos. Os dois últimos valores, referentes aos alunos AI 9 e A20, precisaram ser descartados, pois participaram apenas do pré-teste.

Foi realizado teste de normalidade de Shapiro-Wilk, uma vez que existem menos de 50 observações para análise das variáveis do pré-teste e do pós-teste. Os resultados foram expressos em gráficos, como mostra a Figura 2 (pré-teste) e a Figura 3 (pós-teste). 


\section{Portal Cálculo tem Remédio}

CÁLCULO 竎REMÉDIO Este é um portal de apoio educacional destinado ao ensino do cálculo de dosagem, diluição e gotejamento de medicamento

Aqui no portal você vai encontrar:

\begin{tabular}{|l|}
\hline Ferramentas \\
\hline Apostilas \\
\hline Video Aulas \\
\hline Questões \\
\hline Tutoriais \\
\hline
\end{tabular}

FIGURA 1 - Imagem da tela principal do portal educacional

Fonte: Próprio autor

Teste de normalidade - variável Pós Teste Normal

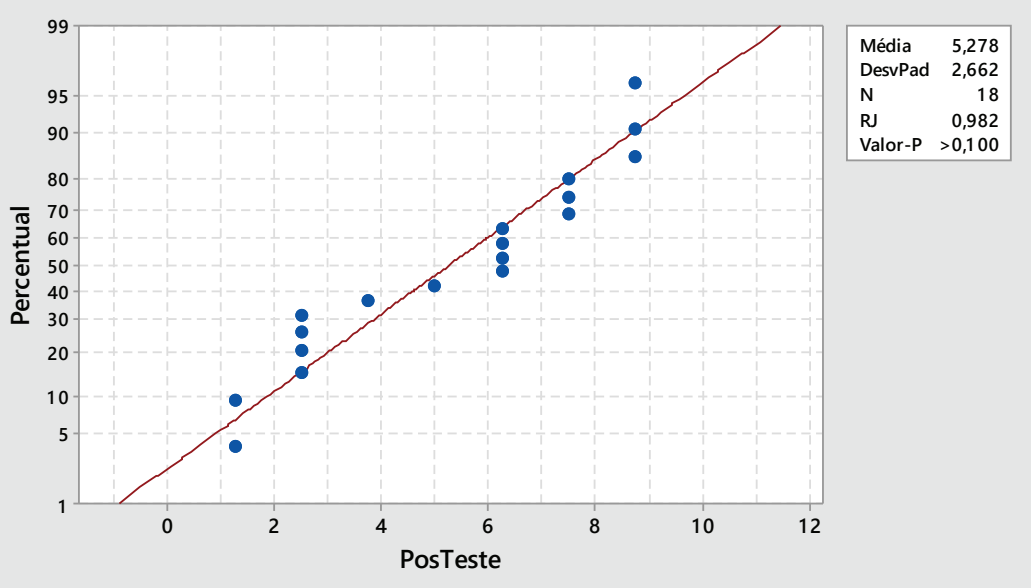

FIGURA 2 - Gráfico da análise de normalidade do rendimento dos alunos no pré-teste Fonte: Próprio autor

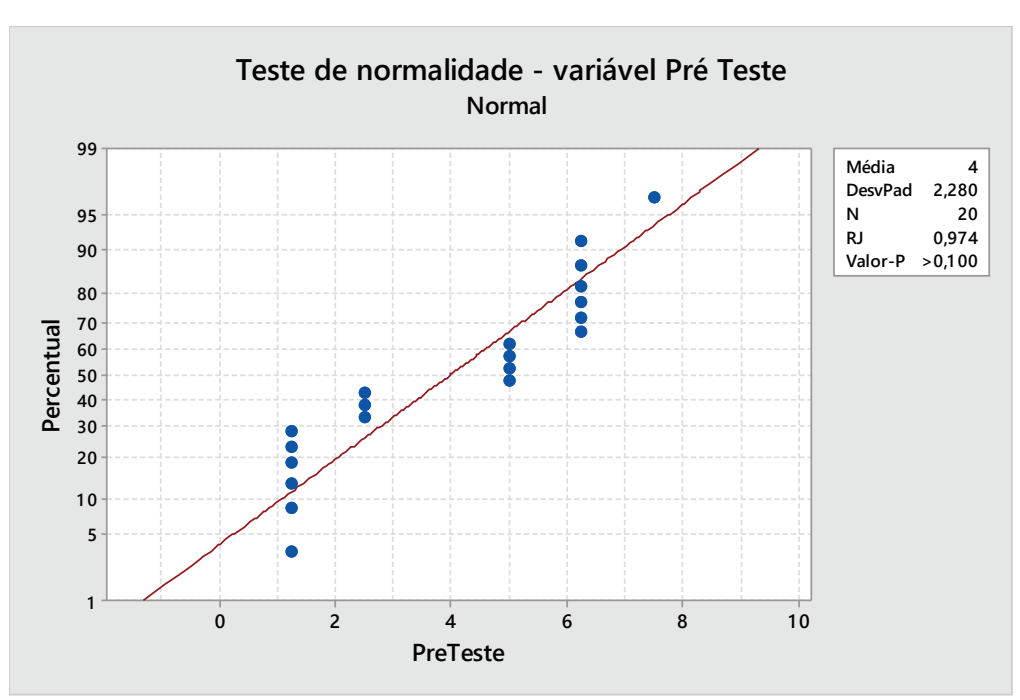

FIGURA 3 - Gráfico da análise de normalidade do rendimento dos alunos no pós-teste Fonte: Próprio autor 


\section{TABELA 1 - Notas no pré-teste e pós-teste}

\begin{tabular}{|c|c|c|}
\hline \multirow{2}{*}{ ALUNO } & \multicolumn{2}{|c|}{ Notas } \\
\hline & Pré teste & Pós teste \\
\hline $\mathrm{A} 1$ & 7,5 & 6,25 \\
\hline A2 & 6,25 & 7,5 \\
\hline A3 & 6,25 & 7,5 \\
\hline A4 & 6,25 & 8,75 \\
\hline A5 & 6,25 & 8,75 \\
\hline $\mathrm{A} 6$ & 6,25 & 8,75 \\
\hline A7 & 6,25 & 7,5 \\
\hline A8 & 5 & 6,25 \\
\hline A9 & 5 & 6,25 \\
\hline A10 & 5 & 6,25 \\
\hline A11 & 5 & 5 \\
\hline A12 & 2,5 & 3,75 \\
\hline A13 & 2,5 & 2,5 \\
\hline $\mathrm{A} 14$ & 2,5 & 1,25 \\
\hline A15 & 1,25 & 1,25 \\
\hline A16 & 1,25 & 2,5 \\
\hline A17 & 1,25 & 2,5 \\
\hline A18 & 1,25 & 2,5 \\
\hline A19 & 1,25 & -- \\
\hline A20 & 1,25 & --- \\
\hline
\end{tabular}

Fonte: próprio autor

Verificou-se que os valores de $p$, em ambos os casos, foram maiores que o nível de significância de $5 \%(0,05)$, e pode-se dizer que os rendimentos dos alunos seguem a distribuição normal.

Foi realizado teste de homocedasticidade de Levene para as duas variâncias, pré-teste e pós-teste, e os resultados foram expressos em gráfico, como mostram as figura 4 .

Observou-se que o valor de $p$ foi maior que o nível de significância de $5 \%(0,05)$, o que indica que os rendimentos dos alunos são homocedásticos, ou seja, as variâncias são iguais.

Satisfeito o pressuposto de normalidade e homocedasticidade, pode-se proceder com a análise de comparação das médias das duas amostras. Utilizou-se o teste $T$ de Student Pareado para comparar médias de duas amostras dependentes.

\section{Teste T Pareado e IC: PreTeste; PosTeste}

Teste T pareado para PreTeste-PosTeste

N Média DesvPad EP Média

PreTeste $18 \quad 4,306 \quad 2,196 \quad 0,517$

\section{PosTeste $\quad 18 \quad 5,278 \quad 2,662 \quad 0,627$ \\ $\begin{array}{lllll}\text { Diferença } & 18 & -0,972 & \mathrm{I}, 098 & 0,259\end{array}$}

IC de $95 \%$ para a diferença média: $(-I, 5 \mid 8 ;-0,426)$

Teste $T$ de diferença de média $=0$ (versus $\neq 0$ ): Valor $T=-3,76$ Valor-p $=0,002$

Observa-se diferença significativa $(p<0,05)$ entre as médias do pré-teste e pós-teste, o que pode ser confirmado no gráfico Boxplot expresso na figura 5.

\section{DISCUSSÃO}

Quando a informática tem seu uso adequado nas escolas, pode possibilitar muitos benefícios aos alunos, como agilidades de lidar com problemas, habilidades de pesquisa, habilidade para novos pensamentos, aumenta a inteligência, abre oportunidades, oferece aos alunos a possibilidade de obter novas informações e conhecer um novo mundo, conhecer a várias culturas, conhecer história de populações antigas e ser novo personagem da escrita da sua própria história na sociedade ${ }^{(13)}$.

$O$ ensino da informática também modifica 0 aluno como indivíduo, pois os alunos tornam-se mais pensantes, críticos, criativos, esforçados, independentes, e pos- 


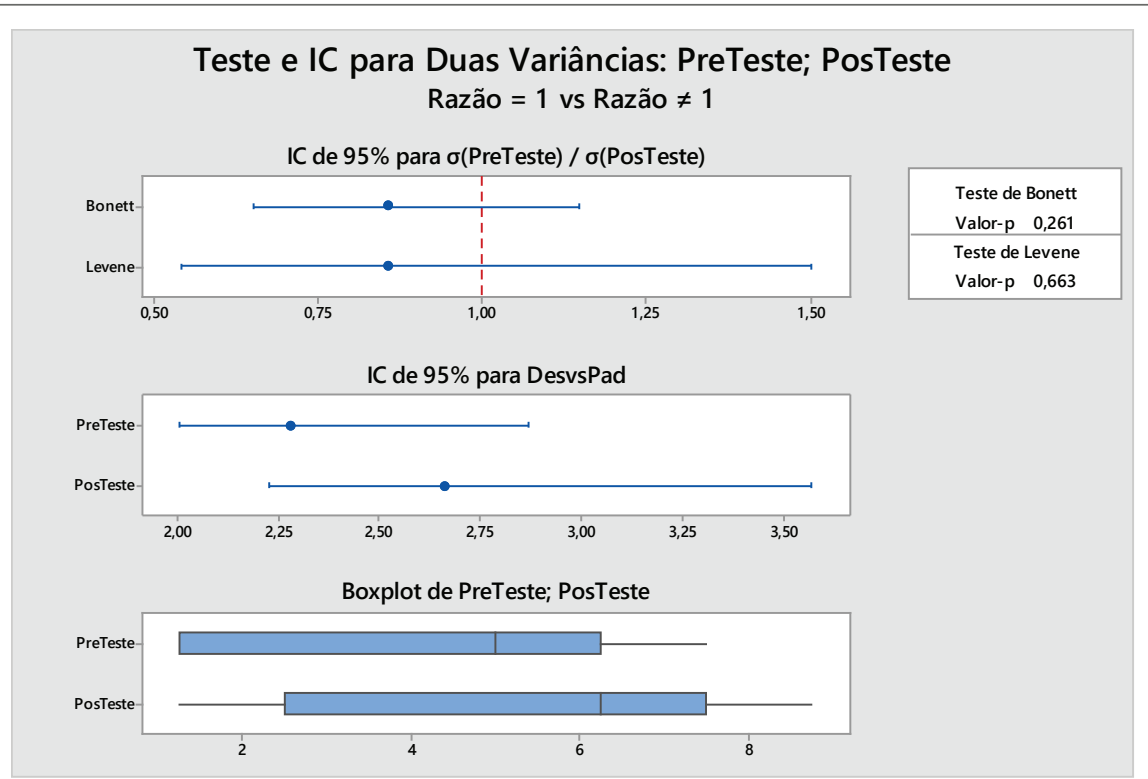

FIGURA 4 - Gráfico do teste para as duas variâncias

Fonte: Próprio autor

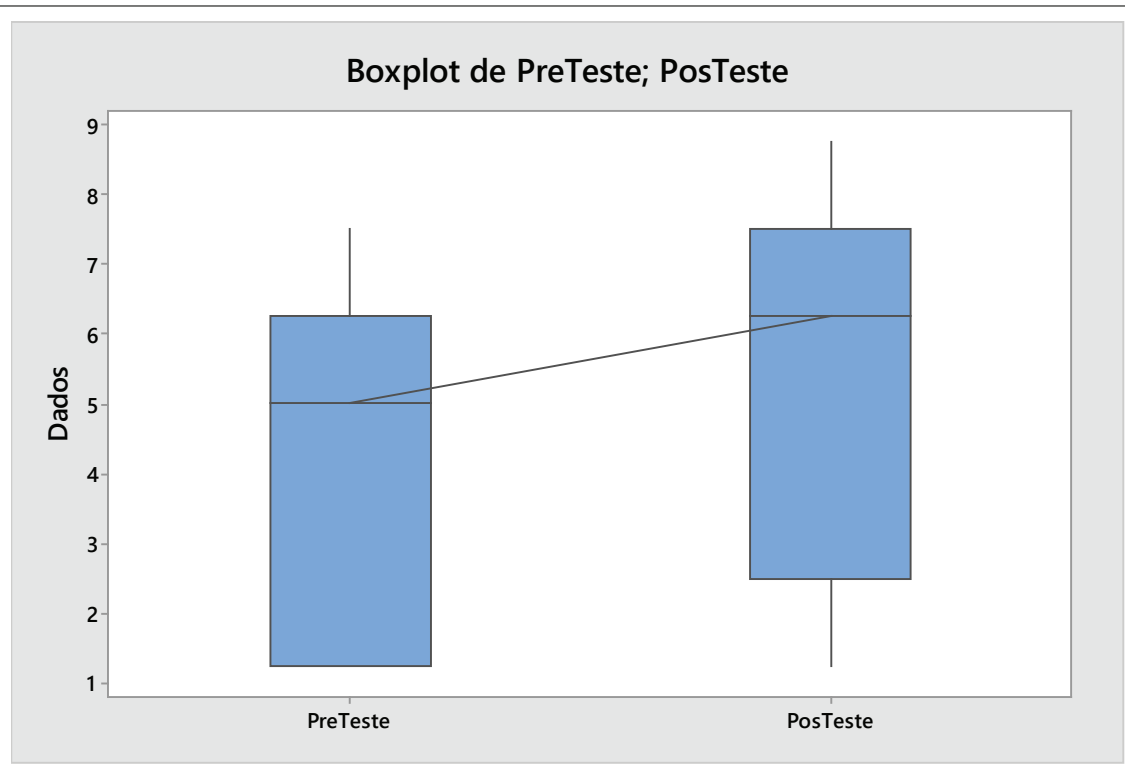

FIGURA 5 - Gráfico Boxplot de pré teste e pós teste

Fonte: Próprio autor

suem habilidades de interagir e conhecer pessoas de forma constante e continuada, bem como outras várias habilidades que os alunos podem obter com a inclusão da informática nasescolas brasileiras.

A pertinência científica e social das tecnologias da educação (TE) produzidas na enfermagem é evidente, pois além de gerar novos conhecimentos, as TE visam a atender demandas e necessidades sociais que emergem de inúmeros contextos, contribuindo para o alargamento do pensar-fazer da Enfermagem enquanto ciência em construção ${ }^{(14)}$.

Para que a formação tecnológica realmente aconteça, é necessário que todos os envolvidos no processo ensino-aprendizagem participem, com responsabilidade de caminharem juntos, como descrito nos " 4 pilares da educação": aprender a conhecer, isto é adquirir os instrumentos da compreensão; aprender a fazer, para poder agir sobre o meio envolvente; aprender a viver juntos, a fim de participar e cooperar com os outros em todas as atividades humanas; e, finalmente, aprender a ser, via essencial que integra as três precedentes ${ }^{(15)}$.

Neste sentido, acreditamos que atualizações e os exercícios enriqueçam a educação na graduação em enfermagem através do uso de programas de computador, baseado nas validações satisfatórias e na avaliação geral favorávelà aplicação desse recurso tecnológico no ensino de Enfermagem.

Os resultados satisfatórios da amostragem estudada contribuem para que a reflexão da sociedade acadêmica, 
pois demonstrou que estratégias inovadoras para estudantes e professores possibilitam a superação de problemas, como desmotivação, escassez de tempo e diferenças no ritmo de aprendizagem entre estudantes. Por fim, a avaliação de materiais educativos pelos usuários pode encorajá-los a construir e usar outros recursos inovadores na educação em enfermagem ${ }^{(11)}$.

\section{CONSIDERAÇÕES FINAIS}

A realização deste estudo possibilitou apresentar um processo de construção e validação de material educativo fundamentado na relação entre as necessidades dos discentes e o conhecimento científico sobre a temática cálculo de medicação.

A metodologia empregada mostrou-se capaz de subsidiar a elaboração de uma tecnologia educativa atrativa e compreensiva, o que pode facilitar a elaboração de outras tecnologias educativas, tanto nesta temática, como em qualquer outra que envolva a necessidade do exercício de exatidão. A pesquisa experimental com o portal logrou êxito quanto aos objetivos propostos e confirmou a hipótese ensejada de que a construção e disponibilização de um portal de apoio educacional para o ensino de matemática aplicada ao cálculo de dosagem de medicamentos podem contribuir para a formação do profissional da área de enfermagem, reduzindo os riscos de dosagens incorretas aplicadas a pacientes.

Dada a simplicidade, qualidade e precisão do produto desenvolvido, estima-se que os resultados futuros são promissores e novas pesquisas podem ser aventadas, disponibilizadas a um número maior de usuários.

Este estudo ficou longe de esgotar o tema sobre as possibilidades e as limitações do uso de software educativos no ambiente de formação do discente de enfermagem. Mas, nunca é demais salientar que os softwares educativos podem ser uma importante ferramenta para o processo de aprendizagemdos discentes, se utilizados de forma contextualizada com os conteúdos trabalhados em sala deaula e com objetivos claramente estabelecidos pelos professores e equipe pedagógica da universidade. 


\section{REFERÊNCIAS}

I.SOUSA, R. P, FILOMENA M. C, MOITA S. C, CARVALHOA. B. G.. Organizadores Tecnologias Digitais na Educação. Tecnologias digitais na educação. -Campina Grande: EDUEPB, $2011.276 \mathrm{p}$

2. ANDRADE, R. S de. SAMPAIO, M. E de C e S. Fundamentos matemáticos para o Curso Técnico em Enfermagem. In: XXV Congresso de Matemática Aplicada e Computacional. Curitiba, 2002.

3. SILVA, J R S. Enfermagem e dificuldades em cálculos aritméticos. Revista Nursing, São Paulo, v. 8I, n. 8, p. 91-98, fev. 2005.

4. COREN - SP CONSELHO REGIONAL DE ENFERMAGEM. Boas práticas: cálculo seguro: Cálculo e diluição de medicamentos. Volume I. São Paulo; 201 I.

5. SILVA M. N,ARAÚJO J. L, FERNANDES N. C. S, OLIVEIRA J. D, SILVA R.T.S, NASCIMENTO E. G. C. Erros de enfermagem e segurança do paciente: o conhecimento de graduandos de enfermagem. Revista Enfermagem Atual. < http://revistaenfermagematual.com.br/revistas/revista_I8-0I.pdf> [periódico na Internet]. jan-fev-mar 20 I7;[acesso 3 abril 20I8].

6. FREITAS F.V, REZENDE FILHO L.A. Modelos de comunicação e uso de impressos na educação em saúde: uma pesquisa bibliográfica. Interface Comun Saúde Educ. 20I I; I5(36):243-55.

7. BERALDINELLI L.M, GUEDES N. A, RAMOS J.P, SILVA M.G. Tecnologia educacional Como Estratégia de Empoderamento de Pessoas Com Enfermidades Crônicas. Rev. Enferm UERJ. 20I4;22(5):603-9.

8. OLIVEIRA, C. C; COSTA, J.W.; MOREIRA, M. Ambientes informatizados de aprendizagem. In: COSTA, J.W.; OLIVEIRA,
M.A. M. (orgs.) Novas linguagens e novas tecnologias: Educação e sociabilidade. Petrópolis:Vozes, 2004.

9. MINITAB. MINITAB QUALITY COMPANION. 2005: MINITAB: Statistical software. Disponível em: < http://www.minitab.com >.Acesso em: out/20 I I.

I0. VASCONCELOS C.T, PINHEIRO A.K, CASTELO A.R, COSTA L.Q, OLIVEIRA R.G. KNOWLEDGE, attitude and practice related to the pap smear test among users of a primary health unit. Rev Latino Am Enfermagem. 20II;19(I):97-105.

I I. GÓES. F. S. N, FONSECA. L. M. M, FURTADO M. C. C, LEITE A.M.SCOCHI C. G.S.Avaliação do objeto virtual de aprendizagem" "Raciocínio diagnóstico em enfermagem aplicado aoprematuro”. Rev. Latino-Am. Enfermagem. [periódico na Internet]. jul-ago 20 I I [acesso 24/I0/20 I2]; I9(4):[08telas]. Disponível em: http://www.scielo.br/pdf/rlae/v19n4/pt_07

12. ALVAREZ A.G, DAL SASSO G. T. M. Aplicação de Objeto Virtual de aprendizagem, para avaliação simulada de dor aguda, em estudantes de enfermagem. Rev. Latino-Am.Enfermagem. [periódico na Internet]. mar-abr20I I;[acesso 3 jan 20II]; I9(2):[09 telas]. Disponível em:http://www.scielo.br/pdf/rlae/v19n2/pt_02

I3. LOPES, M. C. L. P. Formação tecnológica: um fenômeno em foco. Campo Grande: UCDB, 2005.

14. TEIXIRA E, SABOIA V. M. Educação em saúde: tecnologias educacionais em foco. I $^{\text {a }}$ edição. São Caetano do Sul, SP.Difusão editora, 20I I.

15. DELORS, J. Educação: um tesouro a descobrir. 7 ed. São Paulo: Cortez, 2005. 